T. ADATI

KODAI MATH. J.

4 (1981), 327-343

\title{
SUBMANIFOLDS OF AN ALMOST PRODUCT RIEMANNIAN MANIFOLD
}

\author{
By TyuzI AdATI
}

§0. Introduction. When a Riemannian manifold $\bar{M}$ admits a tensor field $F$ of type $(1,1)$ such that $F^{2}=I$ ( $F$ is non-trivial), $\bar{M}$ is called an almost product Riemannian manifold. Let $M$ be a submanifold of an almost product Riemannian manifold $\bar{M}$. We denote by $T_{p}(M)$ the tangent space of $M$ at $P \in M$ and by $T_{p}(M)^{\perp}$ the normal space of $M$ at $P$. If $F T_{p}(M) \subset T_{p}(M)$ for any point $P \in M$, then $M$ is called an invariant submanifold. If $F T_{p}(M) \subset T_{p}(M)^{\perp}$ for any point $P$, then $M$ is called an anti-invariant submanifold. In this paper, we shall study non-invariant, invariant and anti-invariant submanifolds of an almost product Riemannian manifold.

In $\S 1$ and $\S 2$, we obtain for later use fundamental formulas for submanifolds of an almost product Riemannian manifold $\bar{M}$. In $\S 3$, we study hypersurfaces of an almost product Riemannian manifold $\bar{M}$. In $\S 4$ and $\S 5$, we mainly investigate non-invariant submanifolds of $\bar{M}$. We devote $\S 6$ to the study of invariant submanifolds of $\bar{M}$. In the last $\S 7$, we consider anti-invariant submanifolds of $\bar{M}$.

$\S 1$. An almost product Riemannian manifold. Let $\bar{M}$ be an almost product Riemannian manifold of dimension $m$. Then, by definition, there exist a non-trivial tensor field $F$ of type $(1,1)$ and a positive definite Riemannian metric $G$ satisfying

$$
F^{2}=I, \quad G(F \bar{X}, F \bar{Y})=G(\bar{X}, \bar{Y}), \quad \bar{X}, \bar{Y} \in \mathscr{X}(\bar{M}),
$$

where $I$ is the identity and $\mathscr{X}(\bar{M})$ is the Lie algebra of vector fields on $\bar{M}$. It is well known that

$$
G(F \bar{X}, \bar{Y})=G(\bar{X}, F \bar{Y}),
$$

that is, $\Phi$ is symmetric, where $\Phi(\bar{X}, \bar{Y})=G(F \bar{X}, \bar{Y})$.

Let $M$ be an $n$-dimensional manifold immersed in $\bar{M}(m-n=s)$ and $\imath_{*}$ the differential of the immersion $i$ of $M$ into $\bar{M}$. The induced Riemannian metric $g$ of $M$ is given by

$$
g(X, Y)=G\left(i_{*} X, \imath_{*} Y\right), \quad X, Y \in \mathscr{X}(M),
$$

where $\mathscr{X}(M)$ is the Lie algebra of vector fields on $M$. Let $\left\{N_{1}, N_{2}, \cdots, N_{s}\right\}$ be an orthonormal basis of the normal space $T_{P}(M)^{\perp}$ at a point $P \in M$.

Received March 25, 1980 
The transform $F i_{*} X$ of $X \in T_{P}(M)$ by $F$ and $F N_{\imath}$ of $N_{\imath}$ by $F$ can be respectively written in the next form :

$$
\begin{aligned}
& F i_{*} X=\imath_{*} f X+\sum_{\imath=1}^{s} u_{i}(X) N_{\imath}, \quad X \in \mathscr{X}(M), \\
& F N_{\imath}=\imath_{*} U_{i}+\sum_{\jmath=1}^{s} \lambda_{\imath j} N_{\jmath},
\end{aligned}
$$

where $f, u_{\imath}, U_{\imath}$ and $\lambda_{\imath}$ are respectively a linear transformation, 1-forms, vector fields and functions on $M$. Using (1.1) and (1.2),

$$
g(f X, Y)=G\left(i_{*} f X, \imath_{*} Y\right)=G\left(F i_{*} X, \imath_{*} Y\right)=G\left(\imath_{*} X, F i_{*} Y\right)=G\left(\imath_{*} X, \imath_{*} f Y\right) .
$$

Therefore we have $g(f X, Y)=g(X, f Y)$. Furthermore, from $G\left(F i_{*} X, N_{\imath}\right)=$ $G\left(i_{*} X, F N_{\imath}\right)$ and $G\left(F N_{\imath}, N_{j}\right)=G\left(N_{\imath}, F N_{j}\right)$, we can respectively get the equations

$$
\begin{gathered}
u_{i}(X)=g\left(X, U_{\imath}\right), \quad X \in \mathscr{X}(M), \\
\lambda_{\imath \jmath}=\lambda_{j i} .
\end{gathered}
$$

LEMMA 1.1. In submanifold $M$ of an almost product Riemannian manifold $\bar{M}$,

$$
\begin{gathered}
f^{2} X=X-\sum_{\imath=1}^{s} u_{i}(X) U_{\imath} \text { or } f^{2}=I-\sum_{\imath=1}^{s} u_{i} \otimes U_{\imath}, \\
u_{i}(f X)+\sum_{j=1}^{s} \lambda_{\imath j} u_{j}(X)=0, \quad X \in \mathscr{X}(M), \\
f U_{i}+\sum_{j=1}^{s} \lambda_{\imath j} U_{j}=0, \\
u_{j}\left(U_{\imath}\right)=\delta_{j i}-\sum_{k=1}^{s} \lambda_{j k} \lambda_{k \imath} .
\end{gathered}
$$

Proof. From (1.2),

$$
F^{2} i_{*} X=F\left(\imath_{*} f X+\sum_{\imath} u_{i}(X) N_{\imath}\right)=\imath_{*}\left(f^{2} X+\sum_{\imath} u_{i}(X) U_{\imath}\right)+\sum_{\jmath}\left\{u_{j}(f X)+\sum_{\imath} \lambda_{\imath j} u_{i}(X)\right\} N_{\jmath} .
$$

Since $F^{2} i_{*} X=i_{*} X$, we get (1.4) and (1.5). Similarly,

$$
F^{2} N_{\imath}=\imath_{*}\left(f U_{i}+\sum_{j} \lambda_{\imath j} U_{j}\right)+\sum_{k}\left(u_{k}\left(U_{\imath}\right)+\sum_{j} \lambda_{\imath j} \lambda_{j k}\right) N_{k} .
$$

Thus we get (1.6) and (1.7).

(1.5) and (1.6) are equivalent.

Using (1.2), for $X, Y \in \mathfrak{X}(M)$,

$$
G\left(F i_{*} X, F i_{*} Y\right)=G\left(i_{*} f X, \imath_{*} f Y\right)+G\left(\sum_{\imath} u_{\imath}(X) N_{\imath}, \sum_{j} u_{j}(Y) N_{j}\right)
$$




$$
=g(f X, f Y)+\sum_{i} u_{i}(X) u_{i}(Y),
$$

from which

$$
g(f X, f Y)=g(X, Y)-\sum_{\imath} u_{i}(X) u_{\imath}(Y)
$$

§2. A locally product Riemannian manifold. We denote the covariant differentiation in $\bar{M}$ by $\bar{\nabla}$ and the covariant differentiation in $M$ determined by the induced metric on $M$ by $\nabla$. Then the Gauss and Weingarten formulas are respectively given by

$$
\begin{aligned}
& \bar{\nabla}_{\imath * X} \imath_{*} Y=\imath_{*} \nabla_{X} Y+\sum_{\imath=1}^{s} h_{i}(X, Y) N_{\imath}, \quad X, Y \in \mathscr{X}(M), \\
& \bar{\nabla}_{\imath * X} N_{\imath}=-\imath_{*} H_{\imath} X+\sum_{\jmath=1}^{s} \mu_{\imath \jmath}(X) N_{\jmath},
\end{aligned}
$$

where $h_{i}(\imath=1,2, \cdots, s)$ are the second fundamental tensors corresponding to $N_{\imath}$ respectively and $h_{i}(X, Y)=h_{i}(Y, X)$.

Covariantly differentiating $G\left(\imath_{*} Y, N_{\imath}\right)=0$ on $M$,

$$
G\left(\bar{\nabla}_{\imath_{*} \imath_{*}} Y, N_{\imath}\right)+G\left(\imath_{*} Y, \bar{\nabla}_{\imath_{*} X} N_{\imath}\right)=0,
$$

from which $h_{i}(X, Y)=g\left(H_{\imath} X, Y\right)$. Similarly, covariantly differentiating $G\left(N_{\imath}\right.$, $\left.N_{j}\right)=\delta_{i \jmath}$ on $M$, we have $\mu_{\imath \jmath}(X)+\mu_{\jmath i}(X)=0$.

Next, we consider $\bar{\nabla}_{2 * X} F$.

$$
\begin{gathered}
\left(\bar{\nabla}_{\imath * X} F\right) i_{*} Y=\bar{\nabla}_{\imath * X}\left(F i_{*} Y\right)-F \bar{\nabla}_{\imath * X} \imath_{*} Y \\
=\bar{\nabla}_{\imath * X}\left(i_{*} f Y+\sum_{\imath} u_{i}(Y) N_{\imath}\right)-F\left(\imath_{*} \nabla_{X} Y+\sum_{\imath} h_{i}(X, Y) N_{\imath}\right) \\
=\left\{i_{*}\left(\nabla_{X} f\right) Y-\sum_{\imath} u_{i}(Y) H_{\imath} X-\sum_{\imath} h_{i}(X, Y) U_{i}\right\} \\
\quad+\sum_{\imath}\left\{h_{i}(X, f Y)+\left(\nabla_{X} u_{\imath}\right)(Y)-\sum_{\jmath} \mu_{\imath j}(X) u_{j}(Y)\right. \\
\left.\quad-\sum_{\jmath} \lambda_{\imath j} h_{\jmath}(X, Y)\right\} N_{\imath} .
\end{gathered}
$$

When $\bar{M}$ is a locally product Riemannian manifold, that is, $\bar{\nabla} F=0$ ([2], [5]), we have

LEMMA 2.1. If $\bar{M}$ is a locally product Riemannian manifold, then the next equations hold good:

$$
\begin{gathered}
\left(\nabla_{X} f\right) Y=\sum_{i}\left\{u_{i}(Y) H_{\imath} X+h_{i}(X, Y) U_{i}\right\}, \\
h_{i}(X, f Y)+\left(\nabla_{X} u_{\imath}\right)(Y)-\sum_{j} \mu_{\imath j}(X) u_{\jmath}(Y)-\sum_{j} \lambda_{\imath j} h_{j}(X, Y)=0 .
\end{gathered}
$$


Similarly,

$$
\begin{aligned}
\left(\bar{\nabla}_{\imath * X} F\right) N_{\imath}= & \bar{\nabla}_{\imath * X}\left(F N_{\imath}\right)-F \bar{\nabla}_{\imath * X} N_{\imath} \\
= & \bar{\nabla}_{\imath * X}\left(i_{*} U_{i}+\sum_{\jmath} \lambda_{\imath j} N_{j}\right)-F\left(-i_{*} H_{\imath} X+\sum_{j} \mu_{\imath j}(X) N_{\jmath}\right) \\
=\imath_{*} & \left\{\nabla_{X} U_{i}+f H_{\imath} X-\sum_{\jmath} \mu_{\imath j}(X) U_{j}-\sum_{j} \lambda_{\imath j} H_{\jmath} X\right\} \\
& +\left\{\sum _ { j } \left\{h_{j}\left(X, U_{\imath}\right)+h_{i}\left(X, U_{j}\right)+\nabla_{X} \lambda_{\imath j}+\sum_{k} \lambda_{i k} \mu_{k j}(X)\right.\right. \\
& \left.\quad+\sum_{k} \lambda_{j k} \mu_{k \imath}(X)\right\} N_{j}=0 .
\end{aligned}
$$

Thus we have

LEMMA 2.2. If $\bar{M}$ is a locally product Riemannian manifold, then the next equations hold good:

$$
\begin{gathered}
f H_{\imath} X+\nabla_{X} U_{\imath}-\sum_{j} \mu_{\imath \jmath}(X) U_{j}-\sum_{j} \lambda_{\imath j} H_{\jmath} X=0 \\
h_{j}\left(X, U_{\imath}\right)+h_{i}\left(X, U_{j}\right)+\nabla_{X} \lambda_{\imath j}+\sum_{k} \lambda_{i k} \mu_{k j}(X)+\sum_{k} \lambda_{j k} \mu_{k \imath}(X)=0 .
\end{gathered}
$$

Calculating $\left(\nabla_{X} u_{\imath}\right)(Y)$,

$$
\left(\nabla_{X} u_{\imath}\right)(Y)=\nabla_{X}\left\{u_{i}(Y)\right\}-u_{i}\left(\nabla_{X} Y\right)=\nabla_{X}\left\{g\left(Y, U_{\imath}\right)\right\}-g\left(\nabla_{X} Y, U_{\imath}\right)=g\left(\nabla_{X} U_{\imath}, Y\right) .
$$

Hence, (2.2) and (2.3) are equivalent.

§ 3. Hypersurfaces of an almost product Riemannian manifold. Suppose that $M$ is a hypersurface immersed in an almost product Riemannian manifold $\bar{M}[1],[3]$. In this case, (1.2) and (1.3) are respectively written in the following forms :

$$
F i_{*} X=i_{*} f X+u(X) N, \quad F N=i_{*} U+\lambda N,
$$

where $N=N_{1}, u=u_{1}, U=U_{1}, \lambda=\lambda_{11}$ and $u(X)=g(X, U)$. From Lemma 1.1, we have

$$
\begin{aligned}
& f^{2}=I-u \otimes U, \\
& f U=-\lambda U, \\
& u(U)=1-\lambda^{2}, \quad 0 \leqq \lambda^{2} \leqq 1 .
\end{aligned}
$$

The Gauss and Weingarten formulas are respectively given by

$$
\bar{\nabla}_{\imath * X} i_{*} Y=i_{*} \nabla_{X} Y+h(X, Y) N, \quad \bar{\nabla}_{\imath * X} N=-\imath_{*} H X,
$$

where $h=h_{1}, H=H_{1}$ and $h(X, Y)=g(H X, Y)$.

When $\bar{M}$ is a locally product Riemannian manifold, from Lemma 2.1 and 
Lemma 2.2 we have

$$
\begin{gathered}
\left(\nabla_{X} f\right) Y=u(Y) H X+h(X, Y) U, \\
h(X, f Y)+\left(\nabla_{X} u\right)(Y)-\lambda h(X, Y)=0 \text { or } f H X+\nabla_{X} U-\lambda H X=0, \\
2 h(X, U)+\nabla_{X} \lambda=0 .
\end{gathered}
$$

When $\lambda^{2}=1, U$ is a zero vector. Consequently, $M$ is an invariant hypersurface and $f^{2}=I$. Furthermore, we get $F N=\lambda N$. Thus we have

THEOREM 3.1. In order that $M$ is an invariant hypersurface of an almost product Riemannian manifold $\bar{M}$, it is necessary and sufficient that the normal of $M$ is an eigenvector of the matrix $F$.

THEOREM 3.2. In order that $M$ is an invariant hypersurface of an almost product Riemannian manifold $\bar{M}$, it is necessary and sufficient that the induced structure $(f, g)$ of $M$ is an almost product Riemannian structure excepting the case where $f$ is trivial.

Proof. If $f^{2}=I$, we have $u(X) U=0$. Therefore, we get $u(X) g(U, X)=$ $u(X)^{2}=0$, that is, $u(X)=0$. Hence $M$ is invariant.

In the next place, we consider the case where $M$ is not invariant, that is, $\lambda^{2} \neq 1$. Since eigenvalues of $f$ are \pm 1 and $-\lambda$, we have

$$
\operatorname{Tr}(f)=-\lambda+\text { const. }
$$

When $\lambda=0$, the following equations hold good.

$$
\begin{array}{ll}
f^{2}=I-u \otimes U, & u(U)=1, \\
u(X)=g(X, U), & g(f X, f Y)=g(X, Y)-u(X) u(Y) .
\end{array}
$$

Thus, we get the following theorem [4].

THEOREM 3.3. Let $M$ be a hypersurface of an almost product Riemannian manifold $\bar{M}$. If $F N$ is tangent to $M$, then $M$ admits an almost paracontact Riemannian structure.

THEOREM 3.4 [1]. When $M$ is non-invariant hypersurface of a locally product Riemannian manifold $\bar{M}$, the following conditions are equivalent.
(i) $\nabla_{X} f=0$,
(ii) $M$ is totally geodesic,
(iii) $U$ is parallel in $M$.

Proof. (i) When $\nabla_{X} f=0$, we get from (3.4) $u(Y) H X+h(X, Y) U=0$, from which $u(Y) H X=-h(X, Y) U$. Therefore, for $X, Y, Z \in \mathscr{X}(M)$

$$
u(Y) h(X, Z)=-u(Z) h(X, Y) .
$$


Thus, since $u(Y) h(X, Z)$ is symmetric in $X$ and $Y$,

$$
u(Y) h(X, Z)=u(X) h(Y, Z)=-u(Y) h(X, Z) .
$$

By virtue of $u(U)=1-\lambda^{2} \neq 0$, we get $h(X, Z)=0$, that is, $M$ is totally geodesic. Furthermore, from (3.5) we have $\nabla_{x} U=0$.

(ii) When $h(X, Y)=0$, from (3.4) and (3.5), we have $\nabla_{X} f=0, \nabla_{X} U=0$.

(iii) When $\nabla_{X} U=0$, from (3.5) we have $f H X=\lambda H X$. Therefore $f^{2} H X=$ $\lambda f H X=\lambda^{2} H X$, from which

$$
H X-u(H X) U=\lambda^{2} H X .
$$

Since we have $\lambda=$ const. from (3.3) and $\nabla_{X} U=0$, we find $h(X, U)=u(H X)=0$ from (3.6). Thus we get

$$
H X=\lambda^{2} H X,
$$

from which $H X=0$. Consequently, $\nabla_{X} f=0$.

We denote by $\left\{e_{1}, e_{2}, \cdots, e_{n}\right\}$ an orthonormal basis of the tangent space $T_{P}(M)$ at a point $P \in M$. Then we have

THEOREM 3.5. Let $M$ be a non-invariant hypersurface of a locally product Riemannian manifold $\bar{M}$. If $\sum_{\lambda=1}^{n}\left(\nabla_{e_{\lambda}} f\right) e_{\lambda}=0$ and $\operatorname{Tr}(f)=$ const., then $M$ is minımal.

Proof. Since we have $\lambda=$ const. from $\operatorname{Tr}(f)=$ const., we get $h(X, U)=0$ from (3.6). From (3.4)

$$
\sum_{\lambda}\left(\nabla_{e_{\lambda}} f\right) e_{\lambda}=\sum_{\lambda}\left(u\left(e_{\lambda}\right) H e_{\lambda}+h\left(e_{\lambda}, e_{\lambda}\right) U\right)=0
$$

Consequently,

$$
\sum_{\lambda}\left\{u\left(e_{\lambda}\right) h\left(e_{\lambda}, U\right)+h\left(e_{\lambda}, e_{\lambda}\right) u(U)\right\}=\sum_{\mu=1}^{n} h\left(e_{\mu}, e_{\mu}\right)\left(1-\lambda^{2}\right)=0,
$$

from which $\sum_{\lambda} h\left(e_{\lambda}, e_{\lambda}\right)=0$. Hence $M$ is minimal.

$\S 4$. Submanifolds of an almost product Riemannian manifold (I). We consider a non-invariant submanifold $M$ immersed in an almost product Riemannian manifold $\bar{M}$ and assume that $U_{\imath}(i=1,2, \cdots, s)$ are linearly independent. Consequently we have

$$
\sum_{k}\left(\lambda_{i k}\right)^{2}<1(i=1,2, \cdots, s) \text { and } s \leqq n .
$$

Let $\left\{\bar{N}_{1}, \bar{N}_{2}, \cdots, \bar{N}_{s}\right\}$ be the another orthonormal basis of $T_{P}(M)^{\perp}$ at $P \in M$. We put

$$
\bar{N}_{\imath}=\sum_{l=1}^{s} k_{l i} N_{l}
$$


By means of $G\left(\bar{N}_{\imath}, \bar{N}_{j}\right)=\sum_{l} k_{l \imath} k_{l \jmath}$, we have

$$
\sum_{l=1}^{s} k_{l \imath} k_{l \jmath}=\delta_{i \jmath}
$$

from which

$$
\sum_{h=1}^{s} k_{i h} k_{j h}=\delta_{i j}
$$

Consequently matrix $\left(k_{\imath j}\right)$ is an orthogonal matrix. Thus from (4.1), we have

$$
N_{j}=\sum_{h} k_{j l} \bar{N}_{l} .
$$

Making use of (4.1), equations (1.2) and (1.3) are respectively written in the next forms :

$$
\begin{aligned}
& F i_{*} X=i_{*} f X+\sum_{l} \bar{u}_{l}(X) \bar{N}_{l}, \\
& F \bar{N}_{l}=i_{*} \bar{U}_{l}+\sum_{h} \bar{\lambda}_{l h} \bar{N}_{h},
\end{aligned}
$$

where

$$
\begin{aligned}
& \bar{u}_{l}=\sum_{l} k_{l i} u_{l}, \quad \bar{U}_{\imath}=\sum_{l} k_{l i} U_{l}, \\
& \bar{\lambda}_{l h}=\sum_{\imath, j} k_{i l} \lambda_{\imath j} k_{j h} .
\end{aligned}
$$

From (4.4), we obtain

LEMMA 4.1. Let $M$ be a submanifold of an almost product Riemannian manifold $\bar{M}$. When the orthonormal basis $\left\{N_{i}\right\}$ of $T_{P}(M)^{\perp}$ is transformed to the another orthonormal basis $\left\{\bar{N}_{i}\right\}$ of $T_{P}(M)^{\perp}$, if $U_{\imath}(i=1,2, \cdots, s)$ are linearly independent, then $\bar{U}_{\imath}(i=1,2, \cdots, s)$ are also linearly independent, and vice versa.

It is clear that if a submanifold $M$ of a locally product Riemannian manifold $\bar{M}$ is totally geodesic, then $\nabla_{X} f=0$ is satisfied. Conversely, we have the following

THEOREM 4.2. Let $M$ be a submanifold of a locally product Riemannan manifold $\bar{M}$. If $U_{\imath}(i=1,2, \cdots, s)$ are linearly independent and $\nabla_{X} f=0$, then $M$ is totally geodesic.

Proof. Since we have from (2.1) $\sum_{i}\left\{u_{i}(Y) H_{\imath} X+h_{i}(X, Y) U_{i}\right\}=0$, we get the equation

$$
\sum_{\imath}\left\{u_{\imath}(Y) g\left(H_{\imath} X, Z\right)+h_{i}(X, Y) g\left(U_{\imath}, Z\right)\right\}=0, \quad X, Y, Z \in \mathscr{X}(M),
$$

from which 


$$
\sum_{\imath}\left\{u_{i}(Y) h_{i}(X, Z)\right\}=-\sum_{\imath}\left\{u_{i}(Z) h_{i}(X, Y)\right\}
$$

and consequently

$$
\sum_{i} u_{i}(Y) h_{i}(X, Z)=-\sum_{i} u_{i}(X) h_{i}(Y, Z) .
$$

Therefore $\sum_{i} u_{i}(Y) h_{i}(X, Z)$ is symmetric and at the same time skew-symmetric in $X, Y$. Thus we have

$$
\sum_{\imath} u_{i}(Y) h_{i}(X, Z)=0
$$

Since $U_{\imath}(i=1,2, \cdots, s)$ are linearly independent, we get $h_{i}(X, Z)=0(i=1,2, \cdots, s)$, that is, $M$ is totally geodesic.

In $T_{p}(M)$, we denote by $V\left(U_{\imath}\right)$ an $s$-dimensional vector space spanned by $U_{\imath}(i=1,2, \cdots, s)$ and by $V$ an eigenvector of $f$ perpendicular to the vector space $V\left(U_{\imath}\right)$. Then the following equation holds good:

$$
f V=\rho V,
$$

where $\rho$ is an eigenvalue of $f$. Therefore, $f^{2} V=\rho f V=\rho^{2} V$, from which $\left(I-\sum_{\imath} u_{i} \otimes U_{\imath}\right) V=\rho^{2} V$, that is, $V=\rho^{2} V$. Hence we have $\rho^{2}=1$.

When $s<n$, in $T_{P}(M)$, we denote eigenvectors of $f$, which are perpendicular to $V\left(U_{\imath}\right)$ and mutually orthogonal, by $V_{A}(A=s+1, \cdots, n)$. We put

where $\varepsilon_{A}^{2}=1$.

$$
f V_{A}=\varepsilon_{A} V_{A} \quad(A=s+1, \cdots, n),
$$

Next, if we take an eigenvector $U$ of $f$ in the vector space $V\left(U_{\imath}\right)$, the following equation holds good:

$$
f U=\sigma U,
$$

where $\sigma$ is an eigenvalue of $f$. Since we can put $U=\sum_{\imath} c_{i} U_{\imath}$, from (1.6)

$$
f U=f \sum_{\imath} c_{i} U_{\imath}=-\sum_{i, j} c_{\imath} \lambda_{\imath j} U_{j},
$$

from which $\sum_{\imath} c_{\imath} \lambda_{\imath \jmath}=-\sigma c_{\jmath}$. Therefore, if we denote by $\sigma$ an eigenvalue of $f$ in $V\left(U_{\imath}\right)$, then $-\sigma$ is an eigenvalue of the matrix $\left(\lambda_{\imath j}\right)$. The converse is also true.

LEMMA 4.3. Let $M$ be a submanifold of an almost product Rremannian manfold $\bar{M}$. If $U_{\imath}(\imath=1,2, \cdots, s)$ are linearly independent, then we have

$$
\begin{aligned}
\operatorname{Tr}(f) & =-\operatorname{Tr}\left(\lambda_{\imath j}\right)+\sum_{A} \varepsilon_{A} & & (s<n), \\
& =-\operatorname{Tr}\left(\lambda_{\imath j}\right) & & (s=n),
\end{aligned}
$$

where $\varepsilon_{A}^{2}=1(A=s+1, \cdots n)$. 
Proof. We shall prove the case of $s<n$. Since from (1.6) we have $f U_{\imath}=$ $-\sum_{j} \lambda_{\imath j} U_{\jmath}$, matrices $(f),\left(\lambda_{\imath j}\right)$ and $\left(U_{1} U_{2} \cdots U_{s}\right)$ satisfy the relations

$$
(f)\left(U_{1} U_{2} \cdots U_{s}\right)=\left(U_{1} U_{2} \cdots U_{s}\right)\left(-\lambda_{2 j}\right) .
$$

We define matrices $\tilde{U}, L$ by

$$
\begin{aligned}
& \tilde{U}=\left(U_{1} U_{2} \cdots U_{s} V_{s+1} \cdots V_{n}\right), \\
& L=\left(\begin{array}{cc}
-\lambda_{\imath \jmath} & 0 \\
0 & \varepsilon_{A} \delta_{A B}
\end{array}\right) \text {, }
\end{aligned}
$$

where $\delta_{A A}=1, \quad \delta_{A B}=0 \quad(A \neq B) \quad(A, B=s+1, \cdots, n)$. Then we have $(f) \tilde{U}=\tilde{U} L$. Since $|\tilde{U}| \neq 0$, we have $(f)=\tilde{U} L \tilde{U}^{-1}$. If we denote components of $(f), L, \tilde{U}$ and $\tilde{U}^{-1}$ by $f_{\mu}^{\lambda}, l_{\mu \lambda}, u_{\mu}^{\lambda}$ and $v_{\mu}^{\lambda}$ respectively, then we get

$$
f_{\mu}^{\lambda}=\sum_{\omega, \nu} u_{\omega}^{\lambda} l_{\omega \nu} v_{\mu}^{\nu} \quad(\lambda, \mu, \nu, \omega=1,2, \cdots, n) .
$$

Thus we have

$$
\operatorname{Tr}(f)=\sum_{\mu} f_{\mu}^{\mu}=\sum_{\nu} l_{\nu \nu}=-\sum_{i} \lambda_{i i}+\sum_{A} \varepsilon_{A} .
$$

LEMMA 4.4. Let $M$ be a submanfold of an almost product Riemannian manifold $\bar{M}$. If $U_{\imath}(i=1,2, \cdots, s)$ are linearly independent and $\nabla_{X} f=0$, then $\operatorname{Tr}\left(\lambda_{2 j}\right)=$ const..

Proof. Let $\left\{e_{1}, e_{2}, \cdots, e_{n}\right\}$ be an orthonormal basis of $T_{P}(M)$ and extend $e_{\lambda}$ $(\lambda=1,2, \cdots, n)$ to local vector fields $E_{\lambda}$ which are covariantly constant at $P \in M$. Then at $P \in M$,

$$
\begin{aligned}
\nabla_{X} \operatorname{Tr}(f) & =\nabla_{X} \sum_{\lambda} g\left(f e_{\lambda}, e_{\lambda}\right)=\left\{\sum_{\lambda} \nabla_{X} g\left(f E_{\lambda}, E_{\lambda}\right)\right\}_{P} \\
& \left.=\left\{\sum_{\lambda} g\left(\nabla_{X} f\right) E_{\lambda}, E_{\lambda}\right)+2 \sum_{\lambda} g\left(\nabla_{X} E_{\lambda}, f E_{\lambda}\right)\right\}_{P}=\sum_{\lambda} g\left(\left(\nabla_{X} f\right) e_{\lambda}, e_{\lambda}\right)=0 .
\end{aligned}
$$

Thus we have $\operatorname{Tr}(f)=$ const., from which, $\operatorname{Tr}\left(\lambda_{\imath j}\right)=$ const..

THEOREM 4.5. Let $M$ be a submanifold of a locally product Riemannian manifold $\bar{M}$. If $U_{\imath}(i=1,2, \cdots, s)$ are linearly independent, $\operatorname{Tr}(f)=$ const. and $M$ is totally umbilical, then $M$ is totally geodesic.

Proof. If we put $i=j$ in (2.4), we can get

$$
2 \sum_{\imath} h_{i}\left(X, U_{\imath}\right)+\nabla_{X} \sum_{\imath} \lambda_{i i}+2 \sum_{i, k} \lambda_{i k} \mu_{k i}(X)=0
$$

Since $\lambda_{\imath \jmath}$ is symmetric and $\mu_{\imath \jmath}$ skew-symmetric in $i, \jmath, \sum_{\imath, k} \lambda_{i k} \mu_{k \imath}(X)=0$. And by means of $\operatorname{Tr}(f)=$ const. and (4.6), we have $\sum_{\imath} \lambda_{\imath i}=$ const.. Hence we find 


$$
\sum_{\imath} h_{i}\left(X, U_{\imath}\right)=0 \text {. }
$$

Putting $h_{i}(X, Y)=\sigma_{i} g(X, Y)$ and substituting in the above equation, we have $\sum_{\imath} \sigma_{i} g\left(X, U_{\imath}\right)=0$, from which $\sum_{\imath} \sigma_{i} U_{\imath}=0$. Thus we have $\sigma_{\imath}=0$, that is, $M$ is totally geodesic.

THEOREM 4.6. Let $M$ be a submanifold of a locally product Rremannian manifold $\bar{M}$. If $U_{\imath}(\imath=1,2, \cdots, s)$ are linearly independent, $\sum_{\lambda}\left(\nabla_{e_{\lambda}} f\right) e_{\lambda}=0$ and $\operatorname{Tr}(f)=$ const., then $M$ is minimal.

Proof. If we put $X=Y=e_{\lambda}$ in (2.1), we have

$$
\left(\nabla_{e_{\lambda}} f\right) e_{\lambda}=\sum_{i}\left(u_{i}\left(e_{\lambda}\right) H_{i} e_{\lambda}+h_{i}\left(e_{\lambda}, e_{\lambda}\right) U_{\imath}\right),
$$

from which

$$
\sum_{\lambda}\left(\nabla_{e_{\lambda}} f\right) e_{\lambda}=\sum_{i}\left(H_{i} \sum_{\lambda} u_{i}\left(e_{\lambda}\right) e_{\lambda}+\sum_{\lambda} h_{i}\left(e_{\lambda}, e_{\lambda}\right) U_{\imath}\right)=\sum_{i}\left(H_{i} U_{i}+\sum_{\lambda} h_{i}\left(e_{\lambda}, e_{\lambda}\right) U_{i}\right)=0 .
$$

By means of $\operatorname{Tr}(f)=$ const. and (4.6), we have $\sum_{\imath} h_{\imath}\left(X, U_{\imath}\right)=0$, which was shown in the proof of Theorem 4.5. Therefore $\sum_{i} g\left(H_{\imath} X, U_{\imath}\right)=\sum_{\imath} g\left(H_{i} U_{\imath}, X\right)=0$, from which $\sum_{\imath} H_{i} U_{\imath}=0$. Thus we find

$$
\sum_{\imath} \sum_{\lambda} h_{i}\left(e_{\lambda}, e_{\lambda}\right) U_{i}=0
$$

and consequently $\sum_{\lambda} h_{i}\left(e_{\lambda}, e_{\lambda}\right)=0$. Hence $M$ is minimal.

Next, we consider the case of $\lambda_{\imath \jmath}=\lambda_{i} \delta_{\imath \jmath}\left(\lambda_{2}{ }^{2}<1\right)$. In this case, since from (1.7) we have

$$
u_{j}\left(U_{\imath}\right)=\delta_{j i}-\lambda_{j} \lambda_{i} \delta_{j i},
$$

$U_{\imath}(\imath=1,2, \cdots, s)$ are mutually orthogonal. Consequently these vectors are linearly independent. Furthermore, since from (1.6) we have $f U_{i}=-\lambda_{i} U_{\imath}, U_{\imath}$ is an eigenvector of $f$ and $-\lambda_{2}$ is the corresponding eigenvalue of $f$.

Thus we have

THEOREM 4.7. Let $M$ be a submanifold of a locally product Rremannian manifold $\bar{M}$. If $\lambda_{\imath \jmath}=\lambda_{i} \delta_{i j}\left(\lambda_{\imath}{ }^{2}<1\right)$ and $\nabla_{X} f=0$, then $M$ is totally geodesic.

Similarly, in Theorem 4.5 and Theorem 4.6, we can replace the condition that $U_{2}(i=1,2, \cdots, s)$ are linearly independent by $\lambda_{\imath \jmath}=\lambda_{i} \delta_{i j}\left(\lambda_{2}{ }^{2}<1\right)$.

Especially, we put $\lambda_{\imath \jmath}=0$, that is, $u_{j}\left(U_{\imath}\right)=\delta_{j i}$. In this case, $F N_{\imath}$ is tangent to $M$. Since we have $f^{3} X=f X$ by means of $f U_{\imath}=0$, we obtain the following

THEOREM 4.8. In a submanifold $M$ of an almost product Riemannian manifold 
$\bar{M}$, if $F N_{\imath}(i=1,2, \cdots, s)$ are tangent to $M$, then the induced structure tensor $f$ satısfies $f^{3}-f=0$.

It is obvious that the following theorems hold good.

THEOREM 4.9. In a submanifold $M$ of a locally product Riemannian manifold $\bar{M}$, if $F N_{\imath}(\imath=1,2, \cdots, s)$ are tangent to $M$ and $\nabla_{X} f=0$, then $M$ is totally geodesic.

THEOREM 4.10. In a submanifold $M$ of a locally product Rremannan manifold $\bar{M}$, if $F N_{\imath}$ is tangent to $M$ and $M$ is totally umbilical, then $M$ is totally geodesic.

THEOREM 4.11. In a submanifold $M$ of a locally product Riemannian manifold $\bar{M}$, if $F N_{\imath}$ is tangent to $M$ and $\sum_{\lambda}\left(\nabla_{e_{\lambda}} f\right) e_{\lambda}=0$, then $M$ is minimal.

Furthermore, we assume $s=n$. Since $f U_{i}=0$ and $U_{\imath}(\imath=1,2, \cdots, n)$ are linearly independent, we get $f=0$. Thus we have

THEOREM 4.12. In an n-dimensional submanifold $M$ of a locally product Riemannain manifold $\bar{M}$ of dimension $2 n$, if $F N_{\imath}$ is tangent to $M$, then $M$ is anti-invariant and totally geodesic.

§5. Submanifolds of an almost product Riemannian manifold (II). In this section, we assume that $U_{\imath}(\imath=1,2, \cdots, s)$ are not always linearly independent. Let $\left\{N_{1}, N_{2}, \cdots, N_{s}\right\},\left\{\bar{N}_{1}, \bar{N}_{2}, \cdots, \bar{N}_{s}\right\}$ be orthonormal bases of the normal space $T_{P}(M)^{\perp}$. If we put

$$
\bar{N}_{\imath}=\sum_{l} k_{l i} N_{l}
$$

then the matrix $\left(k_{\imath \jmath}\right)$ is an orthogonal matrix and we have (4.2), (4.3), where

$$
\begin{gathered}
\bar{u}_{\imath}=\sum_{l} k_{l i} u_{l}, \quad \bar{U}_{\imath}=\sum_{l} k_{l i} U_{l}, \\
\bar{\lambda}_{l h}=\sum_{i, j} k_{i l} \lambda_{\imath} k_{j h} .
\end{gathered}
$$

By means of (5.2), if $U_{\imath}(\imath=1,2, \cdots, s)$ are linearly dependent, then $\bar{U}_{\imath}$ $(i=1,2, \cdots, s)$ are also linearly dependent. And the greatest number of the linearly independent vector fields in $U_{\imath}(i=1,2, \cdots, s)$ is invariant under the transformation (5.1).

Furthermore, because $\lambda_{\imath \jmath}$ is symmetric in $\imath$ and $\jmath$, from (5.3), we can find that under a suitable transformation (5.1) $\lambda_{\imath \jmath}$ reduces to $\bar{\lambda}_{\imath j}=\lambda_{i} \delta_{i j}$, where $\lambda_{\imath}$ $(i=1,2, \cdots, s)$ are eigenvalues of $\left(\lambda_{\imath j}\right)$. In this case, we have $\bar{u}_{j}\left(\bar{U}_{\imath}\right)=\delta_{j i}-\lambda_{j} \lambda_{i} \delta_{j i}$, that is,

$$
\bar{u}_{\imath}\left(\bar{U}_{\imath}\right)=1-\lambda_{\imath}{ }^{2}, \quad \bar{u}_{j}\left(\bar{U}_{\imath}\right)=0 \quad(\imath \neq j),
$$

and 


$$
f \bar{U}_{\imath}=-\lambda_{i} \bar{U}_{\imath} \text {. }
$$

For $h$ such as $\lambda_{h}{ }^{2}=1, \bar{U}_{h}$ is a zero vector and $F \bar{N}_{h}=\lambda_{h} \bar{N}_{h}$. Consequently $\bar{N}_{h}$ is an eigenvector of $F$. When $\lambda_{l}{ }^{2} \neq 1(l=1,2, \cdots, p \leqq s), \bar{U}_{l}(l=1,2, \cdots, p)$ are linearly independent, because these vectors are mutually orthogonal.

Therefore $F i_{*} X, F \bar{N}_{\imath}$ can be written as follows :

$$
\begin{gathered}
F i_{*} X=i_{*} f X+\sum_{l=1}^{p} \bar{u}_{l}(X) \bar{N}_{l}, \quad(p \leqq \min (s, n)), \\
\left\{\begin{array}{l}
F \bar{N}_{l}=\imath_{*} \bar{U}_{l}+\lambda_{l} \bar{N}_{l} \quad(l=1,2, \cdots, p),(p<s, p \leqq n), \\
F \bar{N}_{h}=\quad \lambda_{h} \bar{N}_{h} \quad(h=p+1, \cdots, s),
\end{array}\right.
\end{gathered}
$$

respectively, where $\lambda_{l}{ }^{2} \neq 1(l=1,2, \cdots, p)$ and $\lambda_{h}{ }^{2}=1(h=p+1, \cdots, s)$. Especially, when $p=s$, in place of (5.7) the following equation holds good:

$$
F \bar{N}_{l}=i_{*} \bar{U}_{l}+\lambda_{l} \bar{N}_{l}, \quad \lambda_{l}{ }^{2} \neq 1 \quad(l=1,2, \cdots, s) .
$$

LEMMA 5.1. Let $M$ be a submanifold of an almost product Riemannian manifold $\bar{M}$. A necessary and sufficient condition for $U_{\imath}(i=1,2, \cdots, s)$ to be linearly independent is that at every point of $M$ normals are not the eigenvector of $F$.

Proof. We consider the condition for $U_{\imath}(\imath=1,2, \cdots, s)$ to be linearly dependent. Let $N$ be a unit normal of $M$ which is an eigenvector of $F$. If we put $\bar{N}_{s}=N$ and transform the orthonormal basis $\left\{N_{1}, N_{2}, \cdots, N_{s}\right\}$ of $T_{P}(M)^{\perp}$ to another orthonormal basis $\left\{\bar{N}_{1}, \bar{N}_{2}, \cdots, \bar{N}_{s}\right\}$, then $\bar{U}_{2}(\imath=1,2, \cdots, s)$ are linearly dependent, because $\bar{U}_{s}$ is a zero vector. Consequently, $U_{\imath}(\imath=1,2, \cdots, s)$ are linearly dependent.

Conversely, if $U_{\imath}(i=1,2, \cdots, s)$ are linearly dependent, then by a suitable transformation of $\left\{N_{1}, N_{2}, \cdots, N_{s}\right\}$, we get a zero vector $\bar{U}_{s}$ and the normal $\bar{N}_{s}$ corresponding to $\bar{U}_{s}$ is an eigenvector of $F$. Thus, the lemma was proved.

LEMMA 5.2. In a submanifold $M$ of an almost product Riemannian manifold $\bar{M}$, we have

$$
\operatorname{Tr}(f)=-\sum_{l=1}^{p} \lambda_{l}+\sum_{A=p+1}^{n} \varepsilon_{A} \quad(p<s, p \leqq n),
$$

where $\lambda_{l}(l=1,2, \cdots, p)$ are eigenvalues of $\left(\lambda_{\imath j}\right)$ satisfying $\lambda_{l}{ }^{2} \neq 1$ and $\varepsilon_{A}{ }^{2}=1$ $(A=p+1, \cdots, n)$. Especially, when $p=s$,

$$
\begin{aligned}
\operatorname{Tr}(f) & =-\operatorname{Tr}\left(\lambda_{\imath j}\right)+\sum_{A=s+1}^{n} \varepsilon_{A}, & & \varepsilon_{A}{ }^{2}=1 \quad(s<n), \\
& =-\operatorname{Tr}\left(\lambda_{\imath j}\right) & & (s=n) .
\end{aligned}
$$

or

Proof. We prove the case of $p<s$. From (5.5), we have $f \bar{U}_{l}=-\lambda_{l} \bar{U}_{l}$ $(l=1,2, \cdots, p)$, where $\bar{U}_{l}(l=1,2, \cdots, p)$ are linearly independent. Thus we get 
(5.8).

From Theorem 4.2 and Lemma 5.1, we have

THEOREM 5.3. Let $M$ be a submanifold of a locally product Riemannian manifold $\bar{M}$. If at every point of $M$ normals are not the eigenvector of $F$ and $\nabla_{X} f=0$, then $M$ is totally geodesic.

Similarly, in Theorem 4.5 and Theorem 4.6, the condition that $U_{\imath}(\imath=1,2$, $\cdots, s)$ are linearly independent can be replaced by the condition that at every point of $M$ normals are not the eigenvector of $F$.

THEOREM 5.4. Let $M$ be a submanifold of a locally product Riemannian manifold $\bar{M}$. If $\lambda_{\imath \jmath}=\lambda_{i} \delta_{i j}$, where $\lambda_{l}{ }^{2} \neq 1 \quad(l=1,2, \cdots, p), \lambda_{h}{ }^{2}=1(h=p+1, \cdots, s)$ $(p<s, p \leqq n)$, and $\nabla_{X} f=0$, then $h_{l}(X, Y)=0(l=1,2, \cdots, p)$.

Proof. From (2.1) we have

$$
\sum_{l=1}^{p}\left\{u_{l}(Y) H_{l} X+h_{l}(X, Y) U_{l}\right\}=0 .
$$

Consequently,

$$
\begin{aligned}
\sum_{l} u_{l}(Y) h_{l}(X, Z) & =-\sum_{l} u_{l}(Z) h_{l}(X, Y)=\sum_{l} u_{l}(X) h_{l}(Y, Z) \\
& =-\sum_{l} u_{l}(Y) h_{l}(X, Z) . \quad(X, Y, Z \in \mathscr{X}(M))
\end{aligned}
$$

Thus, we get $\sum_{l} u_{l}(Y) h_{l}(X, Z)=0$, from which $h_{l}(X, Z)=0(l=1,2, \cdots, p)$.

Similarly, when $\lambda_{\imath \jmath}=\lambda_{i} \delta_{i j}$, where $\lambda_{l}{ }^{2} \neq 1(l=1,2, \cdots, p)$ and $\lambda_{h}{ }^{2}=1(h=p+1$, $\cdots, s)(p<s, p \leqq n)$, the following theorems hold good.

If $\operatorname{Tr}(f)=$ const. and $M$ is totally umbilical, then $h_{l}(X, Y)=0(l=1,2, \cdots, p)$.

If $\sum_{\lambda}\left(\nabla_{e_{\lambda}} f\right) e_{\lambda}=0$ and $\operatorname{Tr}(f)=$ const., then $\sum_{\lambda} h_{l}\left(e_{\lambda}, e_{\lambda}\right)=0(l=1,2, \cdots, p)$.

§ 6. Invariant submanifolds of an almost product Riemannian manifold. Suppose that $M$ is an invariant submanifold immersed in an almost product Riemannian manifold $\bar{M}$. Then $U_{\imath}(i=1,2, \cdots, s)$ are zero vector fields and consequently (1.2), (1.3) are respectively written as follows.

$$
F i_{*} X=\imath_{*} f X, \quad F N_{\imath}=\sum_{\jmath} \lambda_{\imath j} N_{\jmath},
$$

where

$$
\sum_{k} \lambda_{j k} \lambda_{k i}=\delta_{j i}
$$

that is $\sum_{k} \lambda_{j k}{ }^{2}=1, \sum_{k} \lambda_{j k} \lambda_{k \imath}=0(\imath \neq j)$. 
Moreover, from (1.4) and (1.8), we get

$$
f^{2}=I, \quad g(f X, f Y)=g(X, Y), \quad X, Y \in \mathfrak{X}(M) .
$$

Hence, $M$ is an almost product Riemannian manifold excepting the case where $f$ is trivial.

LEMma 6.1. If $M$ is an invariant submanifold of an almost product Riemannian manifold $\bar{M}$, the next equations hold good.

$$
\Phi\left(i_{*} X, i_{*} Y\right)=\phi(X, Y), \quad\left(\bar{\nabla}_{{ }^{*} Z} \Phi\right)\left(i_{*} X, i_{*} Y\right)=\left(\nabla_{Z} \phi\right)(X, Y), \quad X, Y, Z \in \mathscr{X}(M),
$$

where $\Phi(\bar{X}, \bar{Y})=G(F \bar{X}, \bar{Y}), \phi(X, Y)=g(f X, Y), \bar{X}, \bar{Y} \in \mathfrak{X}(M)$.

Proof. $\Phi\left(i_{*} X, i_{*} Y\right)=G\left(F i_{*} X, i_{*} Y\right)=G\left(i_{*} f X, i_{*} Y\right)=g(f X, Y)=\phi(X, Y)$.

Next,

$$
\left(\bar{\nabla}_{\imath_{*} Z} \Phi\right)\left(i_{*} X, i_{*} Y\right)=\bar{\nabla}_{\imath * Z}\left(\Phi\left(i_{*} X, i_{*} Y\right)\right)-\Phi\left(\bar{\nabla}_{\imath * Z} \imath_{*} X, \imath_{*} Y\right)-\Phi\left(i_{*} X, \bar{\nabla}_{\imath_{* Z} \imath_{*}} Y\right) .
$$

On the other hand,

$$
\begin{aligned}
& \Phi\left(\bar{\nabla}_{\imath_{*} \imath_{*}} X, i_{*} Y\right) \\
& =\Phi\left(i_{*} \nabla_{Z} X+\sum h_{i}(Z, X) N_{\imath}, \imath_{*} Y\right)=G\left(F i_{*} \nabla_{Z} X+\sum h_{\imath}(Z, X) F N_{\imath}, \imath_{*} Y\right) \\
& =G\left(i_{*} f \nabla_{Z} X, \imath_{*} Y\right)=g\left(f \nabla_{Z} X, Y\right)=\phi\left(\nabla_{Z} X, Y\right) .
\end{aligned}
$$

Therefore

$$
\left(\bar{\nabla}_{\imath_{*} Z} \Phi\right)\left(\imath_{*} X, i_{*} Y\right)=\nabla_{Z}(\phi(X, Y))-\phi\left(\nabla_{Z} X, Y\right)-\phi\left(X, \nabla_{Z} Y\right)=\left(\nabla_{Z} \phi\right)(X, Y) .
$$

THEOREM 6.2. Let $M$ be a submanifold of an almost product Riemannian manifold $\bar{M}$. A necessary and sufficient condition for $M$ to be invariant is that the induced structure $(f, g)$ of $M$ is an almost product Riemannan structure whenever $f$ is non-truvial.

Proof. It is clear that, if $M$ is invariant, then $M$ is an almost product Riemannian manifold whenever $f$ is non-trivial. Conversely, suppose that $M$ with the induced structure $(f, g)$ is an almost product Riemannian manifold. Then, since $\sum_{i} u_{i}(X) U_{\imath}=0$, we get

$$
\sum_{\imath} u_{\imath}(X) g\left(U_{\imath}, X\right)=\sum_{\imath} u_{i}(X)^{2}=0,
$$

from which $u_{i}(X)=0$. Hence $M$ is invariant.

THEOREM 6.3. Let $M$ be a submanifold of an almost product Riemannian manifold $\bar{M}$. A necessary and sufficient condition for $M$ to be invariant is that 
the normal space $T_{P}(M)^{\perp}$ at every point $P \in M$ admits an orthonormal basis consisting of eigenvectors of the matrix $F$.

Proof. Suppose that, by the transformation of the basis $\left\{N_{1}, N_{2}, \cdots, N_{s}\right\}$, $N_{\imath}, \quad U_{\imath}$ and $\lambda_{\imath \jmath}$ was respectively transformed into $\bar{N}_{\imath}, \bar{U}_{\imath}$ and $\lambda_{i} \delta_{i \jmath}, \lambda_{\imath}$ being eigenvalues of $\left(\lambda_{i j}\right)$. If $M$ is invariant, then we have $F \bar{N}_{i}=\lambda_{i} \bar{N}_{\imath}, \lambda_{l}{ }^{2}=1$. Hence $\bar{N}_{\imath}(i=1,2, \cdots, s)$ are eigenvectors of $F$.

Conversely, suppose that $\bar{N}_{\imath}(i=1,2, \cdots, s)$ are eigenvectors of $F$. Then, by virtue of $F \bar{N}_{i}=\lambda_{i} \bar{N}_{\imath}\left(\lambda_{l}{ }^{2}=1\right)$, we obtain $\bar{U}_{i}=0$. Consequently $M$ is invariant.

THEOREM 6.4. If $M$ is an invariant submanifold of a locally product Riemannian manifold $\bar{M}$, then $M$ is a locally product Riemannian manifold whenever $f$ is non-trivial.

Proof. Making use of Lemma 6.1 (or (2.1)), from $\bar{\nabla} F=0$ we can easily obtain $\nabla_{X} f=0$.

THEOREM 6.5 [5]. In a submanifold $M$ of a locally product Riemannian manifold $\bar{M}$, if the equations

$$
\text { (i) }\left\{\begin{array} { l } 
{ F i _ { * } X = i _ { * } X , } \\
{ F N _ { \imath } = - N _ { \iota } }
\end{array} \text { or } \quad \text { (ii) } \left\{\begin{array}{l}
F i_{*} X=-i_{*} X, \\
F N_{\imath}=N_{\iota}
\end{array}\right.\right.
$$

are satisfied, then $M$ is totally geodesic.

Proof. In the case (i), we have $f=I$. Therefore from (2.3) we get $H_{\imath} X=0$. Hence $M$ is totally geodesic.

Similarly, we obtain

THEOREM 6.6. In a submanifold $M$ of a locally product Riemannian manifold $\bar{M}$, if the equations

(i) $\left\{\begin{array}{l}F i_{*} X=\imath_{*} X, \\ F N_{l}=-N_{l}(l=1,2, \cdots, q) \\ F N_{h}=N_{h}(h=q+1, \cdots, s)\end{array}\right.$ or $\quad$ (ii) $\left\{\begin{array}{l}F i_{*} X=-\imath_{*} X, \\ F N_{l}=N_{l}(l=1,2, \cdots, q), \\ F N_{h}=-N_{h}(h=q+1, \cdots, s)\end{array}\right.$

are satısfied, then $h_{l}(X, Y)=0(l=1,2, \cdots, q<s)$.

THEOREM 6.7. Let $M$ be an invariant submanifold of a locally product Riemannian manifold $\bar{M}$. If $M$ is totally umbilical and $\{\operatorname{Tr}(f)\}^{2} \neq n^{2}$ (or equivalently, $f$ is non-trivial), then $M$ is totally geodesic.

Proof. From (2.2), we have $h_{i}(X, f Y)=\sum_{j} \lambda_{\imath j} h_{\jmath}(X, Y)$. If we put $h_{i}(X, Y)$ $=\sigma_{i} g(X, Y)$, we get $\sigma_{i} g(X, f Y)=\sum_{j} \lambda_{\imath}, \sigma_{j} g(X, Y)$. Substituting $X=Y=e_{\lambda}$, we 
find $\sigma_{\imath} \sum_{\lambda} g\left(e_{\lambda}, f e_{\lambda}\right)=n \sum \lambda_{\imath j} \sigma_{\jmath}$, that is,

$$
\operatorname{Tr}(f) \sigma_{\imath}=n \sum_{\jmath} \lambda_{\imath j} \sigma_{\jmath} .
$$

We multiply the above equation by $\lambda_{j i}$ and sum for $i$. Then we have

$$
\operatorname{Tr}(f) \sum_{\imath} \lambda_{j i} \sigma_{\imath}=n \sum_{\imath} \sum_{k} \lambda_{j i} \lambda_{i k} \sigma_{k}=n \sigma_{\jmath}
$$

by virtue of (6.1). Consequently

$$
\sigma_{j}=\frac{1}{n} \operatorname{Tr}(f) \sum_{\imath} \lambda_{j i} \sigma_{\imath} .
$$

Substituting the above equation into (6.2), we have

$$
\{\operatorname{Tr}(f)\}^{2} \sum_{j} \lambda_{\imath j} \sigma_{\jmath}=n^{2} \sum_{j} \lambda_{\imath j} \sigma_{\jmath},
$$

from which $\sum_{j} \lambda_{\imath j} \sigma_{\jmath}=0$. Since $\sum_{\imath} \sum_{j} \lambda_{h \imath} \lambda_{\imath j} \sigma_{\jmath}=\sigma_{h}=0(h=1,2, \cdots, s), M$ is totally geodesic.

§ 7. Anti-invariant submanifolds of an almost product Riemannian manifold. Last we consider an anti-invariant submanifold $M$ immersed in an almost product Riemannian manifold $\bar{M}$. In this case, $F i_{*} X, F N_{2}$ are written as follows:

$$
\begin{aligned}
& F i_{*} X=\sum_{\imath} u_{i}(X) N_{\imath}, \\
& F N_{\imath}=i_{*} U_{i}+\sum_{\jmath} \lambda_{\imath j} N_{\jmath} .
\end{aligned}
$$

And (1.4), (1.6) become

$$
\begin{aligned}
& \sum_{\imath} u_{i} \otimes U_{\imath}=I, \\
& \sum_{j} \lambda_{\imath j} U_{\jmath}=0 .
\end{aligned}
$$

In order that the solution of $u_{\imath}(X)=0(\imath=1,2, \cdots, s)$ does not exist except zero vector, it is necessary and sufficient that the rank of the matrix $\left(U_{1} U_{2} \cdots U_{s}\right)$ is $n$ and consequently $s \geqq n$.

When $s=n, U_{\imath}(\imath=1,2, \cdots, n)$ are linearly independent and we have $\lambda_{\imath \jmath}=0$ from (7.4). Thus we obtain the following theorem by virtue of Theorem 4.2.

THEOREM 7.1. In a locally product Riemannian manifold $\bar{M}$ of dimension $2 n$, an antiinvaruant submanifold $M$ of dimension $n$ is totally geodesic.

When $s>n, U_{\imath}(i=1,2, \cdots, s)$ are linearly dependent. Suppose that, by a suitable transformation (5.1) of the orthonormal basis $\left\{N_{1}, N_{2}, \cdots, N_{s}\right\}, N_{\imath}, U_{\imath}$ and 
$\lambda_{\imath \jmath}$ are transformed to $\bar{N}_{\imath}, \bar{U}_{\imath}$ and $\lambda_{i} \delta_{i}$, respectively, which $\lambda_{\imath}$ are eigenvalues of $\left(\lambda_{\imath j}\right)$. Then, since $(7.4)$ becomes $\lambda_{i} \bar{U}_{i}=0(\imath=1,2, \cdots, s)$, we can assume that $\bar{U}_{l}$ $(l=1,2, \cdots, n)$ are linearly independent, $\bar{U}_{h}(h=n+1, \cdots, s)$ zero vectors, $\lambda_{l}=0$ $(l=1,2, \cdots, n)$ and $\lambda_{h}^{2}=1 \quad(h=n+1, \cdots, s)$. Consequently $\bar{U}_{l}(l=1,2, \cdots, n)$ are unit vectors which are mutually orthogonal and $\bar{N}_{h}(h=n+1, \cdots, s)$ are eigenvectors of $F$.

Now, denote by $\left\{e_{1}, e_{2}, \cdots, e_{n}\right\}$ the orthonormal basis of $T_{P}(M)$. If we put $F i_{*} e_{k}=N_{k}^{\prime}, F i_{*} e_{l}=N_{l}^{\prime}(k, l=1,2, \cdots, n\}$, then $G\left(N_{k}^{\prime}, N_{l}^{\prime}\right)=\delta_{k l}$. Therefore, we can take the normals $\tilde{N}_{l}(l=1,2, \cdots, n)$ such as $F i_{*} e_{l}=\bar{N}_{l}$.

Thus we obtain

THEOREM 7.2. If $M$ is an antl-invariant submanifold of a locally product Riemannian manfold $\bar{M}$, for the normals $N_{l}(l=1,2, \cdots, n)$ corresponding to the orthonormal basis $\left\{e_{1}, e_{2}, \cdots, e_{n}\right\}$ of $T_{P}(M), h_{l}(X, Y)=0(l=1,2, \cdots, n)$.

On a Riemannian product manifold $\bar{M}=\bar{M}_{1} \times \bar{M}_{2}$, K. Yano and M. Kon proved Theorem 7.1 and Theorem $7.2[6]$.

\section{REFERENCES}

[1] T. Adati and T. Miyazawa, Hypersurfaces immersed in an almost product Riemannian manifold II, TRU Math., 14-2 (1978), 17-26.

[2] S. Tachibana, Some theorems on locally product Riemannian spaces, Tôhoku Math. Jour., 12 (1960), 281-292.

[3] M. OKumura, Totally umbilical hypersurfaces of a locally product Riemannian manifold, Kōdai Math. Sem. Rep., 19 (1967), 35-42.

[4] T. Miyazawa, Hypersurfaces immersed in an almost product Riemannian manifold, Tensor (N.S.), 33-1 (1979), 114-116.

[5] K. Yano, Differential geometry on complex and almost complex spaces, Pergamon Press (1965).

[6] K. Yano ANd M. Kon, Submanifolds of Kaehlerian product manifolds, Attı Acc. Naz. dei Lincei, S. VIII-Vol. XV (1979), 267-292. 
